\title{
Multimode STEM Imaging and Tomography of Radial Heterostructure Nanowire Li-Ion Mini-Batteries
}

\author{
V.P. Oleshko ${ }^{1,4}$, T. Lam ${ }^{2}$, D. Ruzmetov ${ }^{2,3}$, A.V. Davydov ${ }^{1}$, S. Krylyuk ${ }^{1,3}$, P. Haney ${ }^{2}$, H.J. Lezec ${ }^{2}$, J. \\ Cumings $^{4}$, A.A. Talin ${ }^{2,5}$
}
${ }^{1}$ Material Measurement Laboratory and ${ }^{2}$ Center for Nanoscale Science and Technology, National Institute of Standards and Technology, Gaithersburg, MD 20899
${ }^{3}$ Institute for Research in Electronics and Applied Physics and ${ }^{4}$ Dept. of Materials Science and Engineering, University of Maryland, College Park, MD 20742
${ }^{5}$ Sandia National Laboratories, Livermore, California 94551, USA

Semiconductor nanowire (NW) devices show the potential for probing the intrinsic reasons for fading of the specific electrode capacity and make possible insights into relationships between electrical transport and structure in Li-ion batteries (LiBs). The effects of collective behavior characteristic of large arrays in real batteries could be eliminated in individual nanobatteries, thus simplifying interpretation of results. The small dimensions allow imaging of the entire NW-LiB in a FESEM and/or S/TEM to observe changes in the microstructure during cycling in a pA range [1]. Such approach may constitute a powerful engineering platform that offers attractive opportunities for nanoscale diagnostics of electrochemical processes involving cross-instrumentation coordination and comparison of imaging, diffraction and spectroscopy modes, assembling of nano-batteries with essential parts transparent to electrons, and FIB-assisted integration on MEMS-based chips for electrical testing in situ. Here, we report on a 3D characterization of all-solid-state mini-LiBs composed of a metallized Si NW core with radially deposited $\mathrm{LiCoO}_{2}$ cathode, lithium phosphorus oxynitride (LiPON) electrolyte and an amorphous Si anode shells $[1,2]$. Single-crystalline $<111>$ Si NWs were utilized as a scaffold for the entire battery. Using a series of detectors operating in selected ranges of collection angles, multiple STEM signals have been acquired for the same (pixel) position (FIG. 1). Various contrast mechanisms and strong contrast variations resulting in complete contrast reversal even in 0.5 to $1.5 \mu \mathrm{m}$-thick NW-LiBs allowed us to visualize internal nanoscale features, i.e., 80 to $120 \mathrm{~nm}$ pinhole-free LiPON layer, buried electrode-electrolyte interfaces, and textured plateletlike 160 to $200 \mathrm{~nm}$-long hexagonal $\mathrm{LiCoO}_{2}$ crystallites ( $\mathrm{R} \overline{3} \mathrm{~m}$ space group) in the orientations that favor the $\mathrm{Li}^{+}$ionic transport from the cathode directly to the current collector. The STEM offers evident benefits over the broad-beam illumination coherent TEM, which requires samples usually less than $100 \mathrm{~nm}$ in thickness. The advantage is that the HAADF signal generated by incoherent Rutherford-like electrons scattered out to high angles, in which the registered images have different levels of contrast related both to the atomic number (chemical composition) of the sample and the density and thickness (mass-thickness) of the material. In STEM, partial temporal coherence may arise because of the relatively low spread in energies of the illuminating beam if field emission sources used. Contrary to TEM, STEM is not limited by the spherical aberrations of the objective lens, which may cause contrast delocalization. In addition, STEM enables higher contrast and less blurring in specimens at least up to $1.5 \mu \mathrm{m}$ in thickness. Even if diffraction effects are not completely avoidable, Z-contrast in the incoherent HAADF-STEM mode relates the image intensity monotonically to the atomic number, material density and thickness making a much better approximation to a mass thickness image than a coherent BF TEM. Recording datasets of multiple STEM signals, we have utilized correlative multivariate statistical analysis [3] to perform unbiased phase classification (FIG.1, insets). Furthermore, employing high-tilt STEM tomography (FIG. 2 ), we have reconstructed the complex morphology of the $\mathrm{LiCoO}_{2}-\mathrm{LiPON}$ interface and estimated a 
maximum expected discharge capacity of individual NW-LiBs as $0.1-0.2 \mathrm{pAh}$ depending on the available $\mathrm{LiCoO}_{2}$ volume.

References: [1] V.P. Oleshko, et al., Microsc. Microanal., 2011, 17 (Suppl. 2), 1658; ibid, 2012, 18 (Suppl. 2), 1322. [2] D. Ruzmetov et al., Nano Letters., 2012, 12, 505. [3] W. Grogger, F. Hofer, G. Kothleitner, Micron, 1998, 29, 43.



FIG. 1. Multimode STEM montage showing a NW-LiB's internal microstructure. Trivariate histogram of STEM intensities (upper left inset) indicates distinct clusters of the major phases. Composite image of the upper NW-LiB's part (right bottom inset) shows extracted phases and subtracted background (red). 1 - $<111>\mathrm{Si}$ NW, 2- Ti/Pt/Ti metal collector, 3 - LiCoO2 cathode, 4 - LiPON electrolyte layer, 5 - $n$-Si amorphous anode, 6 - supporting polymer net.



FIG. 2. HAADF-STEM tomography of a NW-LiB: (a) Single frame extracted from a tilt-series 3D side (b) and top (c) views of a NW-LiB's tip visualizing segmented $\mathrm{LiPON}$ and $\mathrm{LiCoO}_{2}$ shells around the metallized $\mathrm{Si}$ core and a $\mathrm{LiCoO} 2-\mathrm{LiPON}$ interface (green) where major electrochemical processes occur. The reconstruction reveals a void (7, barely visible in (a) formed during thermal annealing at $700^{\circ} \mathrm{C}$ in oxygen due to the reaction with a metal collector. 